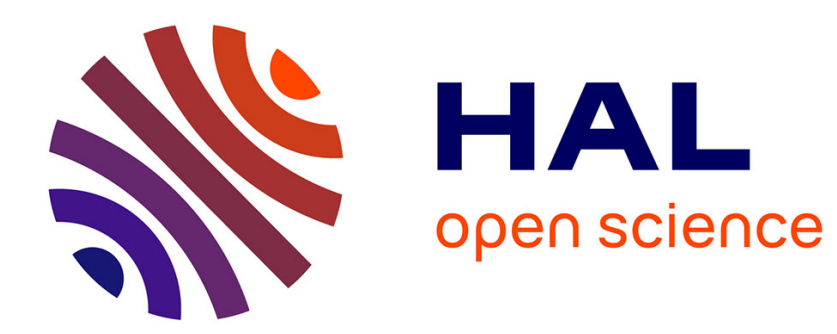

\title{
An Approximate Model for the Adhesive Contact of Viscoelastic Spheres
}

Etienne Barthel, Guillaume Haiat

\section{To cite this version:}

Etienne Barthel, Guillaume Haiat. An Approximate Model for the Adhesive Contact of Viscoelastic Spheres. Langmuir, 2002, 18, pp.9362-70. hal-00001358

\section{HAL Id: hal-00001358 https://hal.science/hal-00001358}

Submitted on 26 Mar 2004

HAL is a multi-disciplinary open access archive for the deposit and dissemination of scientific research documents, whether they are published or not. The documents may come from teaching and research institutions in France or abroad, or from public or private research centers.
L'archive ouverte pluridisciplinaire HAL, est destinée au dépôt et à la diffusion de documents scientifiques de niveau recherche, publiés ou non, émanant des établissements d'enseignement et de recherche français ou étrangers, des laboratoires publics ou privés. 


\title{
An Approximate Model for the Adhesive Contact of Viscoelastic Spheres
}

\author{
E. Barthel*and G. Haiat
}

September 20, 2002

\begin{abstract}
We present a simple model for the adhesive contact of viscoelastic bodies relevant for soft solids. This approach couples the interaction zone creep and the contact zone stress relaxation through a transform of the interaction stresses $g(a)$. We show that $g(a)$, which is proportional to the interaction stress intensity factor, can be extracted from experimentally determined contact variables and at the same time expressed as a function of the interfacial adhesive process. Therefore, the characteristic parameters for adhesion, such as adhesion energy and characteristic tensile stress, for example, can be derived from contact data of viscoelastic bodies. The model also emphasizes the role of stress relaxation within the contact zone in the viscoelastic adhesive contact.
\end{abstract}

keywords: adhesion, crack propagation, contact mechanics, viscoelastic material

\footnotetext{
*Corresponding author: etienne.barthel@saint-gobain.com
} 


\section{Introduction}

\subsection{Adhesive Contact}

The archetypal adhesive contact experiment is the so called JKR test ${ }^{1}$, where a sphere is pressed against a flat plane and force is measured as a function of contact radius for both inward (increasing contact radius) and outward (decreasing contact radius) directions ${ }^{2,3}$. Nanoindentation ${ }^{4}$, surface force microscopy ${ }^{5,6}$ and similar rapidly developing nanoscale technologies are also relevant to the same question. In these cases, however, the contact radius usually eludes detection, so that the contact variables measured are the force and the penetration. To summarize, the quantities accessible experimentally are: 1) the force $F$; 2 ) the penetration $\delta ; 3$ ) the contact radius $a$. We will subsequently call them the macroscopic variables.

The aim of a contact theory is to obtain relations between these macroscopic variables. Three groups of parameters play a role in these relations: 1) the mechanical behaviour of the contacting bodies; 2) their shape; 3) the adhesive process. With a contact theory, numerical values characteristic of the adhesive process can be derived from experimental data, knowing mechanical response and shape. Alternatively, one can predict the behavior of an adhesive contact knowing all three sets of parameters.

\subsection{Previous Viscoelastic Models}

Several papers have recently dealt with theoretical aspects of the adhesive contact between viscoelastic bodies ${ }^{7-10}$. We now recall the main elements of these models.

Contact qualifies the observation that bodies pressed together conform. This happens inside a region called the contact zone (Fig. 1), where a distribution of compressive stresses is generated. In addition, we understand by adhesive processes the interfacial phenomena which generate tensile stresses between surfaces standing at close separation. These adhesive stresses are present not only inside the contact zone, but also outside, whithin the so-called interaction zone, where the surfaces interact but without contact.

The question, for an adhesive contact, is to consistently model conformation inside the contact zone (the inner problem) and adhesive stresses outside (the outer problem). As a result, this problem is generally treated by linear superposition of a modified adhesionless contact solution with a solution to a ring-shaped crack problem ${ }^{11^{-13}}$. In the adhesive contact the adhesive process mirrors the cohesive process in the fracture problem.

The difficulty in the contact problem for viscoelastic bodies is the moving boundary conditions. In the absence of adhesion, the problem was first solved for increasing contact radius only ${ }^{14}$, but proved to be quite a challenge for decreasing contact radius ${ }^{15,16}$. The viscoelastic linear crack problem has been solved by Schapery ${ }^{17-20}$ for both opening and closing cracks. As to the full viscoelastic adhesive contact, Schapery ${ }^{20}$ and Hui et al. ${ }^{21}$ coupled the closing 
viscoelastic crack problem to the viscoelastic contact problem for increasing contact radius. More recently, Lin et al. ${ }^{8}$ proposed a solution (subsequently called LHB) to the inner problem for the receding contact radius case. They came across two "major difficulties" ${ }^{9}: 1$ ) their theory cannot handle the transition between increasing and decreasing contact radius phases 2) they cannot explicitly couple their adhesive contact solution to a crack problem ("the details of the surface forces are not included").

Fourier transform, Sneddon showed ${ }^{22}$, is a powerful method to treat linear elastic contact problems. We have recently applied it to adhesive contacts ${ }^{13}$. More recently still, we extended this method to derive an exact solution to the linear viscoelastic adhesion problem ${ }^{10}$. This model treats the inner and outer problems on the same footing. In the present paper, we now consider the soft solid limit of this model, in which the size of the interaction zone is small compared to the contact zone. The resulting model neatly couples equations typical for both Ting's adhesionless contact ${ }^{16}$ and Schapery's viscoelastic crack models ${ }^{17-20}$. 


\section{Model}

\subsection{Adhesive Elastic Contact}

From earlier work ${ }^{13}$, we introduce the auxiliary functions $g(r)$, which is a suitable transform of the surface stress distribution $\sigma(r)$ (Fig. 1). This transform is defined by (35) in appendix A. Similarly, $\theta(r)$ is a transform, given by (36), of the surface displacement distribution $u_{z}(r)$. These transforms have been chosen so that, under the conditions of linear elastic behavior (Young's modulus $E$, Poisson ratio $\nu$ ) and in the absence of shear stresses at the interface, mechanical equilibrium leads to

$$
g(s)=\mathcal{K} \theta(s) \text { for all } s
$$

where

$$
\mathcal{K}=\frac{E}{2\left(1-\nu^{2}\right)}
$$

Now, the surface displacement is specified inside the contact zone because the surfaces must conform (Fig. 1). Then, $\theta$ is determined inside the contact zone by (36). Furthermore, the surface stress is specified outside the contact zone, inside the interaction zone, by a self-consistent procedure which is discussed in previous papers ${ }^{13}$, and also sketched in appendix B. Then, $g$ is determined outside the contact zone by (35). Finally, under the additional assumption that continuity of the stress distribution at the contact radius $a$ holds, we have continuity of the $\theta$ (and thus $g$ ) functions. For an elastic contact:

$$
g(a)=\mathcal{K} \theta(a) \equiv \mathcal{K}\left(\delta-\delta_{0}(a)\right)
$$

where $\delta$ is the penetration and $\delta_{0}(r)$ is defined by (38) in appendix A. As a result, relation (3) couples interaction stresses (outer problem) through $g$ and penetration (inner problem) through $\theta$. This equation will subsequently be called the coupling equation.

Suppress the adhesive interactions, take $g=0$, and $\delta_{0}(r)$ turns out to be the Hertz (i.e. adhesionless) penetration for the prescribed indenter shape at contact radius $r$. Thus, it readily appears that $\theta(a)$ is this additional flat-punch displacement that, added to the Hertz solution, forms the modified adhesionless contact solution central to the JKR theory ${ }^{1}$ and subsequent contact models $^{12,23}$. In a Maugis-Barquins approach ${ }^{11}$, in terms of fracture mechanics, (3) expresses the cancellation of the inner and outer stress intensity factors. Indeed, $g(a)$ is related in some sense to the stress intensity factor induced by the adhesive stresses: if the interaction zone is small, from (35) one may express the stress intensity factor as a function of $g(a)$ by

$$
K_{\sigma}=\frac{-2 g(a)}{\sqrt{\pi a}} .
$$




\subsection{Viscoelastic Contact}

For a linear viscoelastic material, the equilibrium equation (1) must incorporate the time dependence of the mechanical response. From the usual time convolution of the surface displacement with the stress relaxation function $\psi(t)$, we obtain:

$$
g(t)=\int_{0}^{t} d \tau \psi(t-\tau) \frac{d}{d \tau} \theta(\tau) .
$$

Inversely, with the creep function $\phi(t)$

$$
\theta(t)=\int_{0}^{t} d \tau \phi(t-\tau) \frac{d}{d \tau} g(\tau) .
$$

The response functions $\psi(t)$ and $\phi(t)$ are inverse for the time convolution operator.

Obviously, the response of the system will depend upon its history. It is now necessary to plot the variables as a function of time: a typical contact radius $a$ as a function of time $t$ curve, as considered in the present paper, is displayed on Fig. 2. Note that $\theta(r, t)$ is known in the region below the contact radius curve $(r<a(t))$ while $g(r, t)$ is known - or at least can be determined self-consistently - in the region above $(a(t)<r)$.

We can now generalize the coupling equation (3). For increasing contact radius, we write the continuity of the $\theta$ function at $(\mathrm{a}(\mathrm{t}), \mathrm{t})$ with a combination of $(37)$ and (6):

$$
\delta(t)=\delta_{0}(a(t))+\int_{0}^{t} d \tau \phi(t-\tau) \frac{\partial}{\partial \tau} g(a(t), \tau) .
$$

The time convolution of the $g$ function is depicted by the wavy line in Fig. 2 . Since $g$ is negative, (7) expresses the reduction of the penetration necessary to obtain a given contact radius due to the deformation and creep induced by the attractive interactions. Eq. 7 generalizes (37a) in Ting ${ }^{16}$ to the adhesive case. It is identical to (33) in Hui et al. ${ }^{21}$.

For decreasing contact radius, it is easier to write the continuity of the $g$ function. Thus, we have to determine $g(r, t)$ inside the contact zone $(r<a(t))$. Here, both (5) and (6) are needed to express $g(r, t)$ as a function of the history of $g$ and $\theta$ in the domains where they are known. To wit:

$$
\begin{aligned}
g(r, t)= & \int_{t_{a_{-}}(r)}^{t} d \tau \psi(t-\tau) \frac{\partial}{\partial \tau} \theta(r, \tau) \\
& +\int_{0}^{t_{a_{-}}(r)} d \tau \psi(t-\tau) \frac{\partial}{\partial \tau}\left(\int_{0}^{\tau} d \tau^{\prime} \phi\left(\tau-\tau^{\prime}\right) \frac{\partial}{\partial \tau^{\prime}} g\left(r, \tau^{\prime}\right)\right) .
\end{aligned}
$$

Denoting $t_{\max }$ the time at which the maximum contact radius $a_{\max }$ is reached (Fig. 3), $t_{a_{-}}(r)$ is defined for $r<a_{\max }$ as the inverse to $a(t)$ for $t<t_{\max }$ : for a given $r$, it is the time at which $a(t)=r$ with $t<t_{\max }$. Eq. 8 is valid for both 
increasing and decreasing contact radius. Then, continuity of the $g$ function at time $t$ and radius $a(t)$, for $t>t_{\max }$, reads

$$
g(a(t), t)=\int_{t_{a_{-}(a(t))}}^{t} d \tau \psi(t-\tau) \frac{\partial}{\partial \tau}\left\{\delta(\tau)-\delta_{0}(a(t))\right\}+\bar{g}(a(t), t) .
$$

Eq. 9 is the coupling equation for a decreasing contact radius. It generalizes (40) in Ting ${ }^{16}$ to the adhesive case. It will be compared to the LHB model in the discussion below. The first term on the right-hand side is depicted by the plain wavy line in Fig. 3. The corrective term $\bar{g}$, depicted by the dashed wavy line, is

$$
\bar{g}(a(t), t)=\int_{0}^{t_{a_{-}}(a(t))} d \tau \psi(t-\tau) \frac{\partial}{\partial \tau}\left(\int_{0}^{\tau} d \tau^{\prime} \phi\left(\tau-\tau^{\prime}\right) \frac{\partial}{\partial \tau^{\prime}} g\left(a(t), \tau^{\prime}\right)\right) .
$$

It decreases as $\psi\left(t-t_{a_{-}}(a(t))\right)$. If the stress relaxation is fast, this term will be negligible throughout the outward run, except for the initial stage, just after $t_{\max }$. Then, indeed, $g\left(a_{\max }, t_{\max }\right)=\bar{g}\left(a_{\max }, t_{\max }\right)$, which ensures continuity of the penetration $\delta(t)$ at $t=t_{\max }$.

The adhesive stresses will now be explicitly incorporated in the model. This is actually done in two steps. We first choose a functional form for the spatial distribution of adhesive stresses, which we will call a crack model. Then we specify relations between the parameters which describe the stress distribution so that it approximates a physical adhesive process.

\subsection{Double Hertz Crack Model and Small Interaction Zone}

This crack model is the so called "double-Hertz" approach of Greenwood and Johnson $^{24}$ : for mathematical convenience, an ellipsoidal (Hertz-like) distribution of amplitude $\sigma_{0}$ approximates the attractive stress distribution outside the contact zone. The term "double-Hertz" is somewhat of a misnomer here, since we assume an arbitrary shape for the indentor, and are left with only one Hertz distribution...Assuming the interaction zone extends from $a$ to $c$, we calculate from (35):

$$
g(r)= \begin{cases}\frac{\pi}{4} \sigma_{0} \frac{r^{2}-c^{2}}{\sqrt{c^{2}-a^{2}}} & \text { if } a \leq r \leq c, \\ 0 & \text { if } c<r\end{cases}
$$

Of course, here, $a$ and $c$ are time dependent. As a new assumption, which gives rise to the present simplified version of the viscoelastic adhesive contact, we now suppose that the interaction zone is small, as relevant to soft solids:

$$
\epsilon \equiv c-a \ll a .
$$

Then, to leading order in $\epsilon$,

$$
g(a)=-\frac{\pi}{4} \sigma_{0} \sqrt{2 a \epsilon}
$$


In addition, the time integrals accounting for the adhesive interactions can be treated on a local scale: the interaction radius $c(t)$ is then expanded to first order, and the derivative $d c / d t$ assumed to equal the contact radius velocity $d a / d t$, as in previous works on cracks in viscoelastic media ${ }^{17,18,21}$. We then introduce a fracture dwell time $t_{r}$ defined by

$$
\frac{d a}{d t}=\frac{\epsilon(t)}{t_{r}(t)}
$$

From this small interaction zone assumption, a number of simplifications result in the coupling equations (7) and (9). To leading order, the inward penetration (7) is

$$
\delta(t)=\delta_{0}(a)-\frac{\pi}{4} \sigma_{0} \sqrt{2 a \epsilon} \phi_{0}\left(t_{r}\right)
$$

where

$$
\phi_{0}\left(t_{r}\right)=\frac{1}{t_{r}} \int_{0}^{t_{r}} d \tau \phi\left(t_{r}-\tau\right)
$$

is a momentum of the function $\phi$. Similarly, in (9), we have

$$
\bar{g}(a(t), t) \simeq-\frac{\pi}{4} \sigma_{0} \sqrt{2 a \epsilon} \frac{1}{t_{r}} \int_{t_{i}}^{t_{a_{-}}(a(t))} d \tau \psi(t-\tau) \frac{\partial}{\partial \tau} \int_{t_{i}}^{\tau} d \tau^{\prime} \phi\left(\tau-\tau^{\prime}\right)
$$

with

$$
\begin{aligned}
t_{r} & =t_{r}\left(t_{a_{-}}(a(t))\right), \\
t_{i} & =t_{a_{-}}(a(t))-t_{r} .
\end{aligned}
$$

The total force is obtained from a spatial integration of the $g$ function (cf (39)). The contribution of the interaction zone to the total force is of higher order in $\epsilon$, so that the integration range can actually be restricted to the contact zone. Then,

$$
P=4 \int_{0}^{t} d \tau \psi(t-\tau) \frac{d}{d \tau} \int_{0}^{\min (a(t), a(\tau))} d r\left(\delta(\tau)-\delta_{0}(r)\right)
$$

As with (8), this expression is valid for the inward and outward runs. In the latter case, using (9), we have

$$
\begin{aligned}
P= & 4 \int_{0}^{t_{a_{-}}(a(t))} d \tau \psi(t-\tau) \frac{d}{d \tau} \int_{0}^{a(\tau)} d r\left(\delta(\tau)-\delta_{0}(r)\right) \\
& +4 a(t)\{g(a(t), t)-\bar{g}(a(t), t)\} .
\end{aligned}
$$

Once again, (20) generalizes (42c) in Ting ${ }^{16}$ to the adhesive case. 


\subsection{A Specific Example: Reversible Adhesion}

Any adhesive process can be included in the present theory as long as a model expressing the adhesive stresses as a function of the other variables exist. A rate dependent adhesive process - induced by interdigitation at the interface, for instance - would involve the time derivative of the contact radius $d a / d t$. In principle, the present adhesive contact theory is valid for such an adhesive process, and will couple the dissipation induced by the linear viscoelastic response of the bodies to the intrinsic interfacial dissipation; we can even anticipate that the numerics in the solution would not be much more involved.

Here however, we will consider a simpler adhesive process: reversible adhesion. We thus introduce an adhesion energy $w$. The second parameter which characterizes this adhesive process is the stress amplitude $\sigma_{0}$ introduced earlier. The relevant self-consistency equation is developed in appendix B. For the inward run,

$$
w=\frac{\pi}{8} \sigma_{0}^{2} \epsilon \phi_{1, a}\left(t_{r}\right)
$$

where

$$
\phi_{1, a}\left(t_{r}\right)=\frac{2}{t_{r}^{2}} \int_{0}^{t_{r}} d \tau\left(t_{r}-\tau\right) \phi\left(t_{r}-\tau\right)
$$

is a momentum of the function $\phi$. Similarly, for the outward run,

$$
w=\frac{\pi}{8} \sigma_{0}^{2} \epsilon \phi_{1, r}\left(t_{r}\right)
$$

where

$$
\phi_{1, r}\left(t_{r}\right)=\frac{2}{t_{r}^{2}} \int_{0}^{t_{r}} d \tau \tau \phi\left(t_{r}-\tau\right)
$$

is a different momentum of the function $\phi$. Thus, knowing the adhesion energy $w$ and the typical stress $\sigma_{0}$, one can determine the interaction zone size $\epsilon$, or equivalently (cf (13)) the dwell time $t_{r}$ as a function of crack velocity $d a / d t$. The $\phi_{1}$ functions play the role of effective compliances at the crack tip. Once the crack behavior is determined, it can be coupled to the macroscopic variables through (14) for increasing contact radius, and through (9) along with (16) for decreasing contact radius.

\subsection{Resolution}

\subsubsection{Outline}

We now sketch the resolution of the viscoelastic adhesive contact problem by the present model. Let the penetration history, shape of indentor and viscoelastic response be given. At each time step, we solve a set of two equations with two variables: interaction zone extension $\epsilon$ and dwell time $t_{r}$. The first equation is the self-consistency equation $((21)$ or $(23))$, which treats the crack tip response, and determines the adhesive stress distribution inside the interaction zone. The second is the coupling equation((7) or $(9))$, which links these two local variables 
to the macroscopic variables through $g(a(t), t)$. Then, $\epsilon$ and $t_{r}$ determine the time derivative of the contact radius $a$ through (13). Finally, the contact radius is calculated by integration of this derivative, and the force computed.

If the force is given, the procedure is identical, except that the coupling equation is (19) (or (20) for the outward run). Then the penetration is calculated from (7) or (9). For that purpose, one could be tempted to derive from (9) an explicit expression for $\delta(t)$, in the manner of Ting $\left((41 \mathrm{~b})\right.$ in Ting $\left.{ }^{16}\right)$. Note however that numerically solving the integral equation (9) for $\delta(t)$ is simpler than numerically calculating the explicit expression. The former involves one time-integral $^{25}$, while the latter requires two nested integrals: the price to pay for an explicit expression of $\delta$. Let us also mention that for data treatment, one may always assume that the penetration is the given quantity, even for fixed load experiments: for the same penetration history, the force history will be the same, whether the experiment was done at fixed load or fixed grip (in the stability region). Note also that in practice, the first term in (20) may be derived directly from the force history during the inward phase and the knowledge of the relaxation function, as suggested by Lin et al. ${ }^{8}$.

\subsubsection{Numerical Details}

We here assume that the indenter is a sphere, and that the adhesion model described above (par. 2.4) applies. Then, the standard Maugis ${ }^{12}$ normalization can be used, as detailed in appendix C. The program implemented takes as input an arbitrary pair of viscoelastic functions, provided they are inverse for the convolution integrals (5) and (6). In the following, we assume a normalized standard viscoelastic solid model with creep and relaxation functions given by

$$
\begin{aligned}
\phi(t) & =1+\frac{k}{1-k}(1-\exp (-t)), \\
\psi(t) & =k+(1-k) \exp (-t / k),
\end{aligned}
$$

where $k$ is the characteristic parameter of the normalized creep process. The relaxation and creep functions for $k=0.09$, as assumed below, are illustrated in Fig. 4. Note that for this value of $k$, the relaxed modulus is about ten times smaller than the instantaneous modulus. The adhesion parameter $\lambda$, which is the normalized characteristic stress $\sigma_{0}$, as defined by (49), is 4 , except where specifically mentioned. Due to the local approximation, a difficulty arises at the maximum contact radius $a_{\max }$, where the velocity $d a / d t$ vanishes and the dwell time diverges. This problem is crudely dealt with in the following manner: when the dwell time $t_{r}(t)$ becomes larger than the real time $t$, the algorithm switches to the backward leg (decreasing contact radius). As shown below, this condition is adequate for the numerical parameters assumed for the present calculations. In some cases, it would probably need to be refined by taking into account the interaction zone size in the time cut-off. The differential of the contact radius $a$ was integrated by a Runge-Kutta algorithm ${ }^{25}$, starting from an initial value of 1.5 . 


\subsection{Results}

An example is now given to compare our previous exact ${ }^{10}$ and the present approximate calculations. The penetration history, which is imposed, is given in graph 5 (right-hand scale). The resulting contact radius history, calculated with the full model and the present approximate scheme is shown on the same graph (left-hand scale), and the resulting force, for both models on graph 6 . Satisfactory agreement is found between the two curves, while calculation time dropped by two orders of magnitude. The difference in terms of adherence force between exact and approximate models is less than $10 \%$. The origin of this small discrepancy is in the slightly different maximum contact radii, a difference which may originate from the approximate matching of inward and outward solution at $a_{\max }$ in the simplified model. More generally, both curves exhibit this constant contact radius region typical for the adhesive contact of very viscoelastic systems, to which we refer as the "stick" region ${ }^{10}$. In terms of adherence force, both models give a value around 15. It is noteworthy that this value is in the ratio $1 / k$ with the 1.5 expected in the purely elastic case for such a large $\lambda$, i.e. ten times larger. The "stick" effect and the adherence force enhancement are the most prominent manifestations of contact zone viscoelastic effects, and will be discussed in more details below.

Let us now examine the crack tip effects. The self-consistency relations (21) for the inward run and (23) for the outward run actually provide a one-to-one relation between dwell time (or interaction zone size) and crack velocity. This relation is shown explicitly for the present test values in Fig. 7. We observe that the interaction zone size increases with increasing velocity ${ }^{20}$, due to the larger effective material stiffness, and that the difference between in- and outward cases are minute, as expected ${ }^{7}$. This difference is due to the asymmetry of the direction of motion relative to the crack tip, which is reflected in (21) and (23). To provide further insight into the local variables and their coupling with the macroscopic variables, let us assume an imposed penetration history as given in Fig. 8 (right-hand scale). The calculated interaction zone size $\epsilon$ is displayed on Fig. 8 for $\lambda=4$. It decreases during the inward run, then increases, in accordance with the general behavior of the interaction zone size with contact velocity. A pronounced cusp at $t=2.6$ is due to the approximate matching condition between inward and outward equations at $a_{\max }$ described above. We also plotted $g(a(t))$ on the same graph. In this low penetration example, the variation of the contact radius is weak and according to $(12), g(a(t), t)$ is dominated by the behavior of the interaction zone size, except close to the initial and final stages, where the curvature of the contact radius is more pronounced.

Increasing $\lambda$ from 4 to 8 , the "stick" phenomenon becomes more and more pronounced (Fig. 9): after $t_{\max }$, the contact zone radius flattens, owing to decreasing crack tip velocity ${ }^{20,8}$. The "stick" time however, and the adherence force, are little affected, because $\mathrm{g}(\mathrm{a})$, which determines both, depends only weakly upon $\lambda$ when the interaction zone size is small (cf (53) and (61) or (63)), a result reminiscent of the JKR limit in the elastic adhesive contact. 


\section{Discussion}

\subsection{Structure of the Solution}

We have seen that the viscoelastic adhesive contact model separates into two problems: 1) the viscoelastic crack, which couples the adhesive process with the viscoelastic response; 3 ) the viscoelastic adhesive contact, which couples the viscoelastic contact with the viscoelastic crack.

\subsubsection{The Viscoelastic Crack}

The surface stress generated by the adhesive process inside the interaction zone induce mechanical deformations there. In the small interaction zone size limit, this creep is a purely local process, in which no macroscopic variable is involved, but three local (or "crack") variables: contact radius velocity (or "crack tip" velocity), interaction zone size and dwell time. There is a simple relation (Eq. 13) between these variables. Once the adhesive process and the viscoelastic response are specified, a self-consistent treatment of stress and deformation provides a second relation between two of the local variables, reducing the number of free variables to one, as illustrated in Fig. 7.

The crack tip equation proposed here as an example ((21) for the inward leg and (23) for the outward leg) result from two assumptions: 1) a 'Double-Hertz' crack model 2) a reversible adhesive process, characterized by an adhesion energy $w$ and a stress amplitude $\sigma_{0}$. These equations can be directly compared to Schaperys ${ }^{18,20}$ : for a power law creep compliance, they result in identical expressions within numerical factors of the order unity; for an arbitrary viscoelastic response, they give $\phi_{1}\left(t_{r}\right)$ as another explicit form for Schaperys approximate effective compliance at the crack tip $\phi\left(t_{r} / 3\right)$.

As expected, an alternative local variable is the stress intensity factor $K_{\sigma}$. Indeed, combining (4) and (12), it readily appears that

$$
K_{\sigma}=-\sqrt{\frac{\pi}{2}} \sigma_{0} \sqrt{\epsilon}
$$

The stress intensity factor will thus follow the interaction zone size and decrease with decreasing contact radius velocity. In addition, with (21) or (23), we obtain

$$
w=\frac{K_{\sigma}^{2} \phi_{1}\left(t_{r}\right)}{4}
$$

Such an expression cast the self-consistency equations (21) and (23) into the standard form of an energy release rate (note the factor 2 with similar expressions ${ }^{7}$, due to different definitions of the elastic constant). As such, it emphasizes the meaning of $\phi_{1}\left(t_{r}\right)$ as an effective (creep) compliance.

\subsubsection{Stress Intensity Factor or Adhesion?}

The question may arise as to which is the fundamental quantity that best characterizes the adhesion: the adhesive process ( $w$ and $\sigma_{0}$ in the present example) 
or the stress intensity factor $K_{\sigma}$ ? From the previous discussion, it appears that the adhesive process is more fundamental than the stress intensity factor. The contact radius velocity dependance of the latter (or of the interaction zone size (cf. Fig. 7)) compounds in a single parameter what needs to be known from the adhesive process and the viscoelastic response in order to calculate the impact of the viscoelastic crack on the behavior of the adhesive contact - and only that -. Thus, one can work out the behavior of a viscoelastic adhesive contact with the knowledge of the viscoelastic response and the contact radius velocity dependance of the stress intensity factor. This will require the adhesive contact equations (7) or (9) only. However, if the adhesive process needs to be characterized, then, one has to go through the viscoelastic crack problem and extract the characteristic adhesive parameters from the measured stress intensity factor, using independent knowledge of the viscoelastic functions and self-consistency equations like (21) or (23).

\subsubsection{Adhesive Viscoelastic Contact}

Once the crack behavior is established, one can write down relations between the macroscopic and local variables. In our method, the key element, for inclusion of the crack response, is the value of the $g$ function at $(a(t), t)$. In this respect, it is clear from (12) that $g(a)$ combines both types of variables: local (the interaction zone extension $\epsilon$ ) and macroscopic (the contact radius $a$ ).

For the inward run, the coupling equation (7) actually deals with a 'strain intensity factor' $\theta(a(t), t)$, as expected ${ }^{7}$. For the outward run, the coupling equation (9) states - to put it in fracture mechanics terms - that the stress intensity factor (or more precisely $g(a(t), t)$ ) due to the adhesive interaction stresses equals the time convolution of the penetration with the relaxation function, which is therefore the stress intensity factor due to the contact stresses. Alternatively, (9) states - and it is then the expression of the stress continuity assumption - that the stress generated at $(a(t), t)$ inside the contact zone, by contact between surface and indenter is equal to the stress generated at $(a(t), t)$ outside the contact zone by the adhesive process. This is the reason why the penetration does not depend upon the history of the system before $t_{a_{-}}(t)$ since the final stress state at $r$ depends only upon the period of time when there was contact at $r$. As a further result, the force is independent of the history of the system after $t_{a_{-}}(t)$, because this history (a flat punch displacement, as far as the $r<a(t)$ region is concerned) is summed up in $g(a(t), t)-\bar{g}(a(t), t)$ by $(9)$.

\subsection{Stress Relaxation}

Stress relaxation within the contact zone emerges as the prominent concept in viscoelastic adhesion. It is the key to the apparent fracture energy explored in previous reports ${ }^{7,20}$. In particular, closer inspection shows that (13) and (12) in Greenwood et al. ${ }^{19}$ are limit cases for (19) and (20). Let us now discuss this stress relaxation in more details. It naturally induces global force relaxation: this is evidenced in (20) by the decrease of the first - typically com- 
pressive - term. This term decays approximately as $\psi(t)$. As a result, the force drops sharply when the motion of the indenter is reversed and retraction begins (Fig. 6). However, if the stresses inside the contact zone have relaxed, the contact line cannot recede immediately after reversal of the direction of motion. Indeed, backward motion of the contact line requires the build-up of sufficient tensile stresses within the contact zone, ahead of the crack tip. In the case of viscoelastic bodies, this is achieved only if the pull out motion is sufficiently fast compared with the stress relaxation, as expressed by (9). The result is a time lag between the backward motion of the indenter, as specified by the penetration, and the backward motion of the contact line; a time lag between strain (which controls the penetration) and stress (the crack tip interaction) characteristic of viscoelastic behavior.

As a result, if the relaxation is fast, the compressive stresses relax in proportion to $\psi(\infty)$ while close to rupture, the contact radius velocity is large, so that the tensile stresses are characterized by an effective modulus $\psi\left(t_{r}\right)^{-1} \simeq \psi(0)^{-1}$. Then the effective adhesion energy is enhanced $7,10,19$ by $\psi(0) / \psi(\infty) \simeq 1 / k$ which in our example amounts to the factor of ten mentioned above. Note that for low penetration, the adherence force is weaker than expected from this simple argument. This is because the maximum contact radius is smaller than the equilibrium contact radius for the relaxed modulus, resulting in a reduced adherence force.

\subsection{Comparison with the LHB model}

As previously mentionned, in contrast to the $\mathrm{LHB}^{8}$ equations, the present model is continuous at $t_{\max }$. In addition, the interactions are explicitly included in a form reminiscent of the Schapery crack models. Let us now compare our expression for the stress intensity factor with LHB's. Following these authors, let us introduce

$$
\delta_{1}(t)=\delta\left(t_{a_{-}}(a(t))\right) .
$$

They consider the quantity

$$
\begin{aligned}
\delta(t)- & \delta_{1}(t) \\
= & \int_{0}^{t} d \tau \phi(t-\tau) \frac{\partial}{\partial \tau} \int_{0}^{\tau} d \tau^{\prime} \psi\left(\tau-\tau^{\prime}\right) \frac{\partial}{\partial \tau^{\prime}}\left(\delta(\tau)-\delta_{1}\left(\tau^{\prime}\right)\right) \\
= & -\int_{t_{\max }}^{t} d \tau \phi(t-\tau) \frac{\partial}{\partial \tau} \\
& {\left[g(a(\tau), \tau)-\bar{g}(a(\tau), \tau)+\int_{t_{a_{-}}(a(\tau))}^{\tau} d \tau^{\prime} \psi\left(\tau-\tau^{\prime}\right) \frac{\partial}{\partial \tau^{\prime}} \delta_{1}\left(\tau^{\prime}\right)\right] }
\end{aligned}
$$

where the last expression results from the fact that $\delta(t)-\delta_{1}(t)=0$ for $t<t_{\max }$ and from (9). As a result, for the outward phase, from (34) and (35) in LHB, introducing the so-called reference stress intensity factor, we identify

$$
K_{I}(t)-\psi\left(t-t_{a_{-}}(a(t))\right) \phi(0) K_{I}\left(t_{a_{-}}(a(t))\right)
$$


with

$$
\frac{(2) \psi(0)}{\sqrt{\pi a(t)}} \int_{t_{\max }}^{t} d \tau \phi(t-\tau) \frac{\partial}{\partial \tau}(g(a(\tau), \tau)-\bar{g}(a(\tau), \tau))
$$

where the factor of 2 is from (34) in LHB, and does not appear in (35) in LHB. This quantity $K_{I}$ is quite different from the stress intensity factor as derived from the present theory by (4): typically, the second term in (32) and the $\bar{g}$ term in (33) will decay rapidly. Then,

$$
K_{I}(t)=\frac{(2) \psi(0)}{\sqrt{\pi a(t)}} \int_{t_{\max }}^{t} d \tau \phi(t-\tau) \frac{\partial}{\partial \tau} g(a(\tau), \tau) .
$$

It appears that the LHB stress intensity factor is a sort of mean value of the actual local stress intensity factor $K_{\sigma}$. As such, this quantity is far from intrinsic: in particular, for the same contact radius velocity, its actual value will depend upon the history of the system, instead of being purely local, as it should.

The fundamental reason is that LHB relied on the adhesionless case, where the penetration for the outward leg is expressed as a function of the penetration for the inward leg, $\delta_{1}(t)$. By induction, in the adhesive case, they also considered the quantity $\delta(t)-\delta_{1}(t)$. With the present model, we have shown that the right quantity is actually $\delta(t)-\delta_{0}(a(t))$, which is much simpler. Of course, in the adhesionless case, $\delta_{1}(t)=\delta_{0}(a(t))$ ! 


\section{Conclusion}

The present approach for the viscoelastic adhesive contact treats interaction and contact consistently. Creep in the interaction zone is characterized by an effective compliance which depends upon contact radius velocity. The interaction is then coupled to the penetration or the force through $g(a)$, which depends both upon local crack tip variables (the stress intensity factor $K_{\sigma}$ ) and macroscopic variables (the contact radius $a$ ). In this model, the full contact curve is described continuously, even at the inward to outward transition. The model can be applied to various adhesive processes as long as a constitutive relation between adhesive stresses and other variables in the model is provided. It allows either experimental data analysis or adhesive contact predictions. The results emphasize the role of stress relaxation within the contact zone. In particular, stress relaxation gives rise to: 1) a time lag between retraction of the indenter and contraction of the contact zone ("stick" effect);2) an enhancement of the adherence force.

\section{Acknowledgement}

We thank R. Hengstebeck for linguistic advice. 


\section{A Surface Distributions}

Under the assumption of axial symmetry, we resort to two auxiliary functions $g$ and $\theta$, which are the following transforms of respectively the surface stress $\sigma$ and surface displacement $u_{z}$ distributions:

$$
\begin{aligned}
g(s) & =-\int_{s}^{+\infty} \frac{r \sigma(r)}{\sqrt{r^{2}-s^{2}}} d r, \\
\theta(s) & \equiv \frac{d}{d s} \int_{0}^{s} \frac{r u_{z}(r)}{\sqrt{s^{2}-r^{2}}} d r .
\end{aligned}
$$

In particular, inside the contact zone $(r<a)$, the surface displacement is known through the contact condition

$$
u_{z}(r)=\delta-f(r)
$$

where $\delta$ is the penetration and $f$ the shape of the indentor.

Then, inside the contact zone

$$
\theta(s)=\delta-\delta_{0}(s)
$$

where

$$
\delta_{0}(r)=\frac{d}{d r} \int_{0}^{r} d s \frac{s f(s)}{\sqrt{r^{2}-s^{2}}} .
$$

is the Hertz penetration for a contact radius $s$. Note that this function $\delta_{0}$ is determined by the shape of the indenter only. It is equal to $r^{2} / R$ for a sphere of radius $R$ and to $\frac{\pi}{2} r / \tan \beta$ for a cone of apical angle $\beta$. Finally, the force is

$$
F=4 \int_{0}^{+\infty} \operatorname{drg}(r) .
$$

\section{B Self-consistency}

It is required that the surface stress spatial distribution $\sigma$ be a reasonably faithful representation of the real attractive stress distribution. This is achieved by the so called self-consistency equation. For simplicity, let us here assume that the interaction derives from a potential $V$. Then the adhesion energy

$$
w \equiv V(0)=-\int_{a}^{c} \sigma(r) \frac{d}{d r} h(r)
$$

where $a$ is the contact radius, $c$ the interaction zone radius and the gap $h(r)$ is defined by

$$
h(r)=u_{z}(r)-\delta+f(r) .
$$

In addition, let us assume that inside the interaction zone, the Hertz-like elastic deformation is negligible compared to the deformation induced by the interactions themselves. Then, the gap between the surfaces outside the contact zone is

$$
h(r)=\frac{2}{\pi} \int_{a}^{r} \frac{\theta(s)-\theta(a)}{\sqrt{s^{2}-a^{2}}} d s
$$


and thus an estimate of the thermodynamic adhesion energy can be calculated through (40). Let us here make use of the so-called double Hertz crack model, where the normalized stress distribution outside the contact zone is assumed to be ellipsoidal ${ }^{24}$ :

$$
\sigma(r)=-\sigma_{0} \sqrt{\frac{c^{2}-r^{2}}{c^{2}-a^{2}}} .
$$

Then, one can express the thermodynamic adhesion energy as:

$$
w=\frac{\sigma_{0}}{\sqrt{c^{2}-a^{2}}} \int_{a}^{c} d s \theta^{\prime}(s)(c-s)
$$

This equation ensures that our interaction stress distribution, though not exact, suitably embodies the physics of the adhesive process. In previous works, we have shown that the adhesive contact is relatively insensitive to the functional form of the adhesive stress distribution ${ }^{23}$ : it is only the decay length of the interaction which primarily matters.

It must also be emphasized that self-consistency equations for more complex adhesive phenomena, including velocity dependent phenomena, should be relatively easy to obtain. The simplest one would result from the assumptions that $\sigma_{0}$ is constant, but the effective adhesion energy $w$ depends upon contact radius velocity. The resulting self-consistency equation could be used in the present theory at no extra calculation cost. While a contact radius velocity dependance for $w$ is quite usual, in adhesive models, a constant $\sigma_{0}$ may seem more questionable. However, as shown in the example (par. 2.6), in the large $\sigma_{0}$ limit, the results are weakly dependent upon $\sigma_{0}$. Therefore, the resulting model will probably turn out to be useful.

\section{The Sphere - Normalization}

We specialize our model to the case of the sphere. Thus, following Maugis ${ }^{12}$, we define

$$
K=\frac{8 \mathcal{K}}{3}
$$

where $\mathrm{K}$ is defined in (2). In the viscoelastic case, the normalizing elastic constant is the instantaneous modulus and $K=4 \psi(0) / 3$. We introduce the nor- 
malized quantities

$$
\begin{aligned}
P & =\frac{F}{\pi w R}, \\
A & =\frac{a}{\left(\frac{\pi w R^{2}}{K}\right)^{1 / 3}}, \\
\Delta & =\frac{\delta}{\left(\frac{\pi^{2} w^{2} R}{K^{2}}\right)^{1 / 3}}, \\
\lambda & =\frac{2 \sigma_{0}}{\left(\frac{\pi w K^{2}}{R}\right)^{1 / 3}}, \\
G(S) & =\frac{\pi}{3} \lambda \frac{S^{2}-C^{2}}{\sqrt{C^{2}-A^{2}}}, \\
\tilde{\psi}(t) & =\psi(t) / \psi(0), \\
\tilde{\phi}(t) & =\phi(t) / \phi(0) .
\end{aligned}
$$

Then,

$$
G(A) \simeq-\frac{\pi}{3} \lambda \sqrt{2 A \epsilon}
$$

were $\epsilon=C-A$. The time variables and functions are as defined in section 2 . The inward penetration is

$$
\Delta=\Delta_{0}-\frac{\pi}{3} \lambda \sqrt{2 A \epsilon} \frac{1}{t_{r}} \int_{0}^{t_{r}} d \tau \tilde{\phi}\left(t_{r}-\tau\right)
$$

and the outward penetration is determined by

$$
G(A(t), t)=\int_{t_{a_{-}}(A(t))}^{t} d \tau \tilde{\psi}(t-\tau) \frac{\partial}{\partial \tau}\left(\Delta(\tau)-\Delta_{0}(A(t))+\bar{G}(A(t), t)\right.
$$

where

$$
\bar{G}(A(t), t) \simeq-\frac{\pi}{3} \lambda \sqrt{2 A \epsilon} \frac{1}{t_{r}} \int_{t_{i}}^{t_{a_{-}}(A(t))} d \tau \tilde{\psi}(t-\tau) \frac{\partial}{\partial \tau} \int_{t_{i}}^{\tau} d \tau^{\prime} \tilde{\phi}\left(\tau-\tau^{\prime}\right)
$$

with

$$
\begin{aligned}
t_{r} & =t_{r}\left(t_{a_{-}}(A(t))\right), \\
t_{i} & =t_{a_{-}}(A(t))-t_{r} .
\end{aligned}
$$

In normalized form, and keeping the major terms, the force is

$$
P=\frac{3}{2}\left\{\int_{0}^{t} d \tau \tilde{\psi}(t-\tau) \frac{d}{d \tau}\left(M_{a} \Delta(\tau)-\frac{M_{a}^{3}}{3}\right)-\frac{\pi}{3} \lambda \sqrt{\frac{\epsilon}{2}} \frac{A^{3 / 2}}{3}\right\}
$$


where $M_{a}$ stands for $\min (A(t), A(\tau))$. In normalized form, the self-consistency equations are

$$
\begin{aligned}
1 & =\frac{\pi}{3} \lambda^{2}\left(\frac{d A}{d t}\right) \frac{1}{t_{r}} \int_{0}^{t_{r}} d \tau\left(t_{r}-\tau\right) \tilde{\phi}\left(t_{r}-\tau\right) \\
& =\frac{\pi}{6} \lambda^{2} \epsilon \tilde{\phi}_{1, a}\left(t_{r}\right)
\end{aligned}
$$

for positive $d A / d t$ (inward leg) and

$$
\begin{aligned}
1 & =\frac{\pi}{3} \lambda^{2}\left|\frac{d A}{d t}\right| \frac{1}{t_{r}} \int_{0}^{t_{r}} d \tau \tau \tilde{\phi}\left(t_{r}-\tau\right) \\
& =\frac{\pi}{6} \lambda^{2} \epsilon \tilde{\phi}_{1, r}\left(t_{r}\right)
\end{aligned}
$$

for negative $d A / d t$ (outward leg), where the $\tilde{\phi}_{1}$ are defined as in section 2.4. For small [resp. large] dwell time, the momenta both equal $\tilde{\phi}(0)[$ resp. $\tilde{\phi}(+\infty)]$, from which the elastic self-consistency equation (with the present normalization) is recovered:

$$
1=\frac{\pi}{6} \lambda^{2} \epsilon \tilde{\phi}(l)
$$

where $l=0$ or $l=+\infty$. 


\section{References}

(1) Johnson, K. L.; Kendall, K.; Roberts, A. D. Proc. Roy. Soc. London A, 1971, 324,301 .

(2) Falsafi, A; Tirrell, M.; Pocius, A.Langmuir, 2000, 16, 1816.

(3) Crosby, A. J.; Shull, K. R.; Lin, Y. Y.; Hui, C.-Y.J. Rheol., 2002, 46, 273.

(4) Giri, M.; Bousfield, D. B.; Unertl, W. N.Langmuir, 2001, 17, 2973.

(5) Portigliatti, M.; Koutsos, V.; Hervet, H.; Leger, L.Langmuir, 2000, 16, 6374.

(6) Pickering, J. P. Vancso, G.J. Macromol. Symp., 2001, 166, 189.

(7) Johnson, K. L., in Microstructure and Microtribology of Polymer Surfaces, Tsukruk, V. V. and Wahl, K. J. ed., ACS, Washington D.C., 2000, 24.

(8) Lin, Y. Y.; Hui, C. Y.; Baney, J. M.J. Phys. D: Appl. Phys., 1999, 32, 2250 2260.

(9) Lin, Y. Y.; Hui, C. Y.J. Polym. Sci. B, 2002, 40, 772.

(10) Haiat, G.; Phan Huy, M. C.; Barthel, E.J. Mech. Phys. Solids, in press.

(11) Maugis, D.; Barquins, M.J. Phys. D.: Appl. Phys., 1978, 11, 1989.

(12) Maugis, D.J. Colloid Interface Sci., 1992, 150, 243.

(13) Huguet, A.-S.; Barthel, E.J. Adhesion, 2000, 74, 143.

(14) Lee, E. H.; Radok, J. R. M.J. Appl. Mech., 1960, 27, 438.

(15) Graham, G. A. C.Int. J. Engng Sci., 1965, 3, $27-46$.

(16) Ting, T. C. T.J. appl. Mech., 1966, 33, 845.

(17) Schapery, R. A.Int. Journ. of Fracture, 1975, 11, 141 - 159.

(18) Schapery, R. A.Int. Journ. of Fracture, 1975, 11, 369 - 388.

(19) Greenwood, J. A.; Johnson, K. L.Phil. Mag. A, 1981, 43, $697-711$.

(20) Schapery, R. A.Int. Journ. of Fracture, 1989, 39, 163 - 189.

(21) Hui, C. Y.; Baney, J. M.; Kramer, E. J.Langmuir, 1998, 14, 6570 - 6578.

(22) Sneddon, I. N. Fourier Transforms; Mac Graw Hill, New York, 1951.

(23) Barthel, E.J. Colloid Interface Sci., 1998, 200, 7.

(24) Greenwood, J. A.; Johnson, K. L.J. Phys. D: Appl. Phys., 1998, 31, 3279.

(25) Press, W. H.; Teukolsky, S. A.; Vetterling, W. T.; Flannery, B. P.; Numerical Recipes in C, CUP, New York, 1992. 


\section{Captions}

Fig. 1: schematics of the conceptual division of the adhesive contact into two zones, showing the normal surface stress distribution $\sigma(r)$ (dashed) and the gap between surfaces $h(r)$ (solid line): in the contact zone $(r<a)$, compressive and tensile stresses are found and the gap vanishes; in the interaction zone $(a<r<c)$, the stresses are tensile and the gap opens up. The Double-Hertz model $^{24}$ assumes an ellipsoidal stress distribution in the interaction zone, as shown here.

Fig. 2: schematics for the coupling equation (7) (increasing contact radius), displaying the contact radius $a$ as a function of time on an $(r, t)$ plane. The plane is split into two regions: for $a(t)<r$ the surface stress transform $g(r, t)$ is known, for $r<a(t)$ the surface displacement transform $\theta(r, t)$ is known. The time convolution integral in (7) is depicted here by the wavy line.

Fig. 3: schematics for the coupling equation (9) (decreasing contact radius). The main time integral in (9) is depicted here by the plain wavy line. The dashed line is for the corrective term $\bar{g}$.

Fig. 4: exponential creep (25) and relaxation (26) functions used for all the numerical calculations presented in this paper. The characteristic creep time is the time unit, and the characteristic creep parameter is $k=0.09$.

Fig. 5: imposed penetration (right-hand scale) and resulting contact radius, with $\lambda=4$, for full model ${ }^{10}$ and present approximation. A region where the contact radius is about constant is identified: this "stick" effect is due to the fast relaxation of the stresses inside the contact zone.

Fig. 6: force as function of penetration (same conditions as Fig. 5). Most noteworthy is the ten-fold enhancement of the adherence force over the bare 1.5 expected for a purely elastic system: another effect of stress relaxation.

Fig. 7: dwell time (left-hand, log scale) and interaction zone size (righthand, linear) as a function of log of contact radius (or "crack tip") velocity, for increasing (inward) and decreasing (outward) contact radius, as calculated from the self-consistency equations (21) and (23), which describe the viscoelastic crack tip effect $(\lambda=4)$.

Fig. 8: a lower penetration example: penetration history (top, right-hand scale) and resulting contact radius $a$ (top, left-hand), interaction zone size $\epsilon$ (bottom, left-hand) and $g(a(t), t)$ (bottom, right-hand) for $\lambda=4$, as a function of time. The cusp in the interaction zone size at $t=2.6$ is due to imperfect matching of the in- and outward solutions (cf. section 2.5.2). The stress intensity factor $K_{\sigma}$ is proportional to $\sqrt{\epsilon}$, and has not been plotted here. However, $g(a(t), t)$, which is the link between local ("crack") variables and macroscopic ("contact") variables, and is proportional to $\sqrt{a \epsilon}$, exhibits features from both $a$ and $\epsilon$.

Fig. 9: force (left-hand scale) and contact radius (right-hand) as a function of penetration (same as Fig. 8) and different values of $\lambda$. The "stick" effect (section 2.6) is enhanced for increasing $\lambda$, because the crack tip velocity then decreases. 


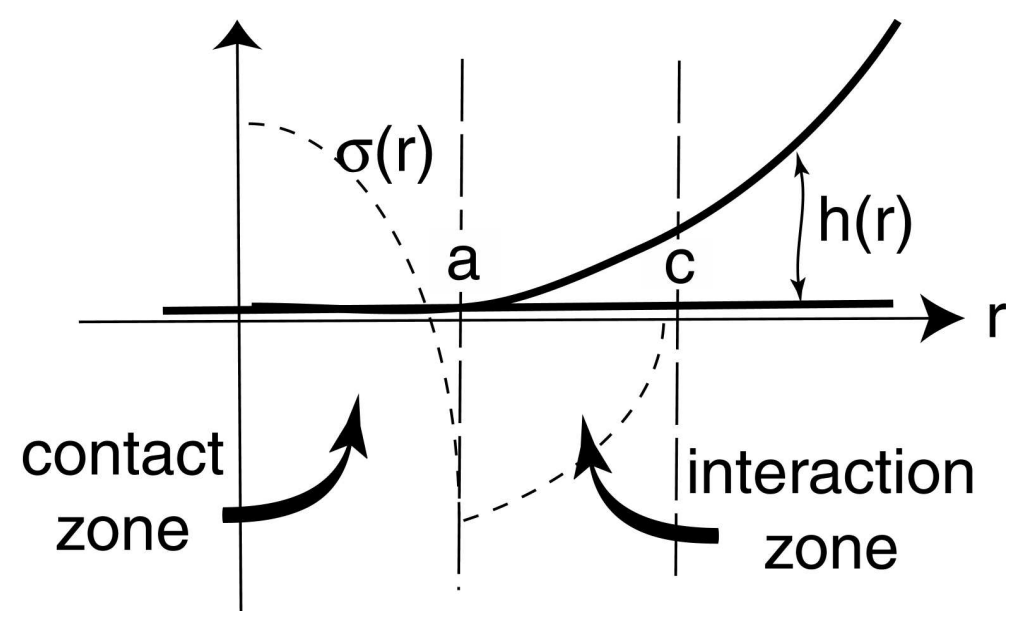

Figure 1: 


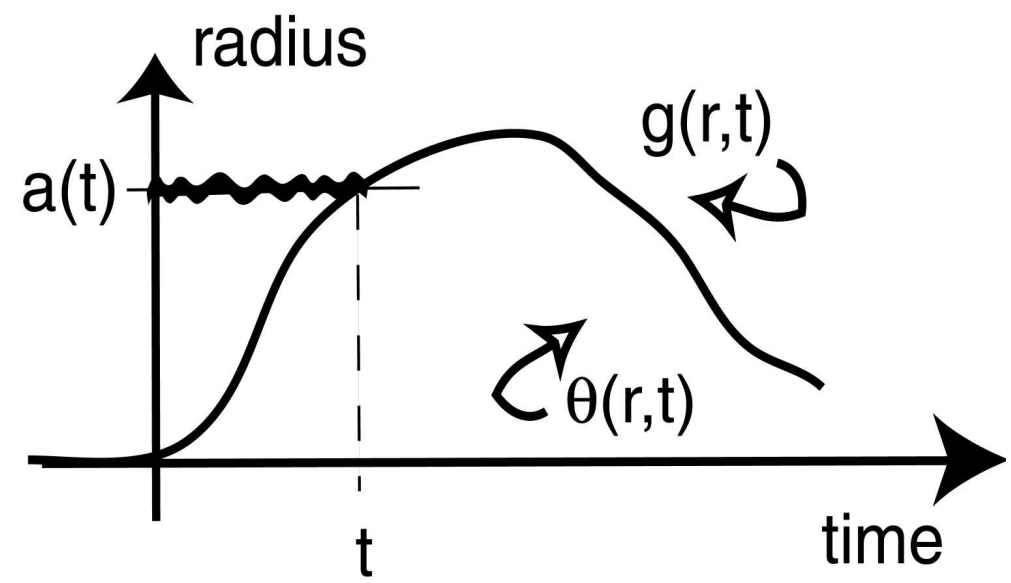

Figure 2:

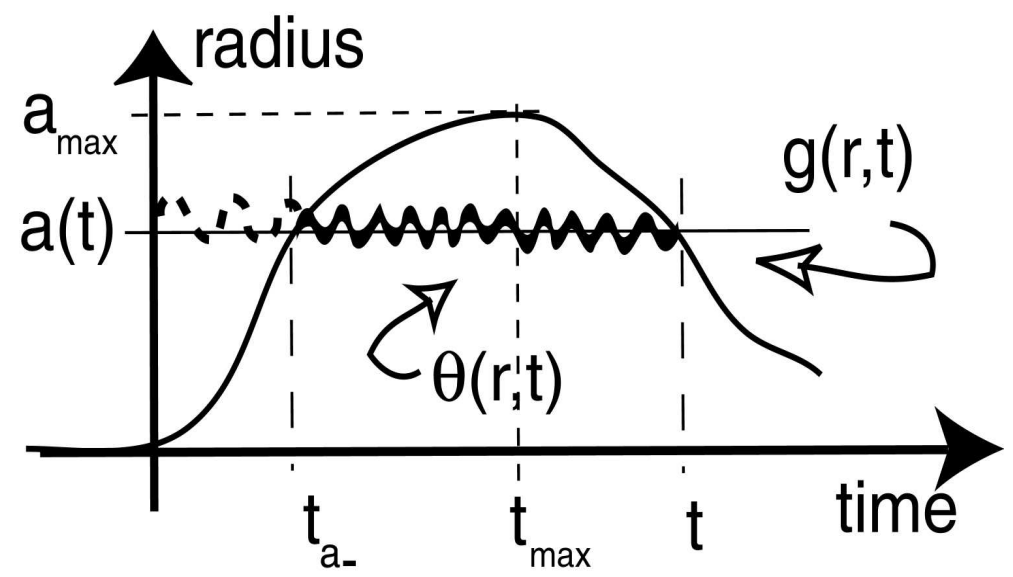

Figure 3: 


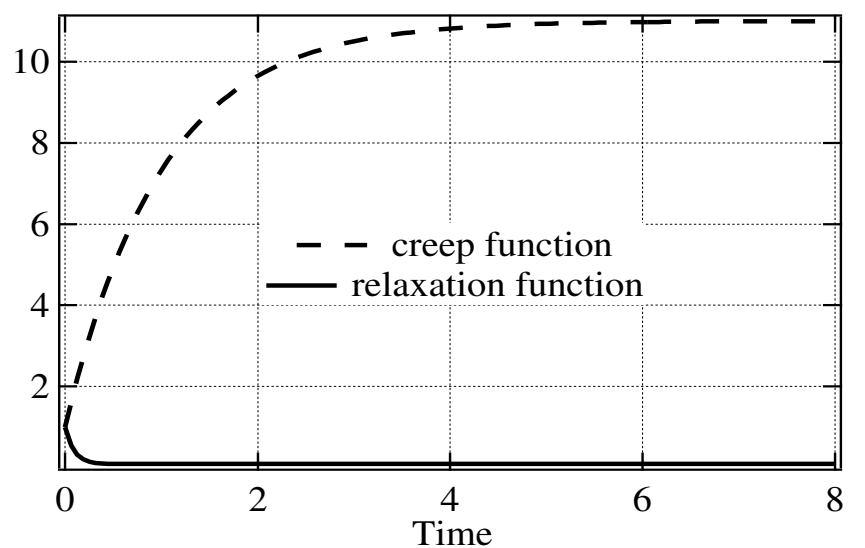

Figure 4:

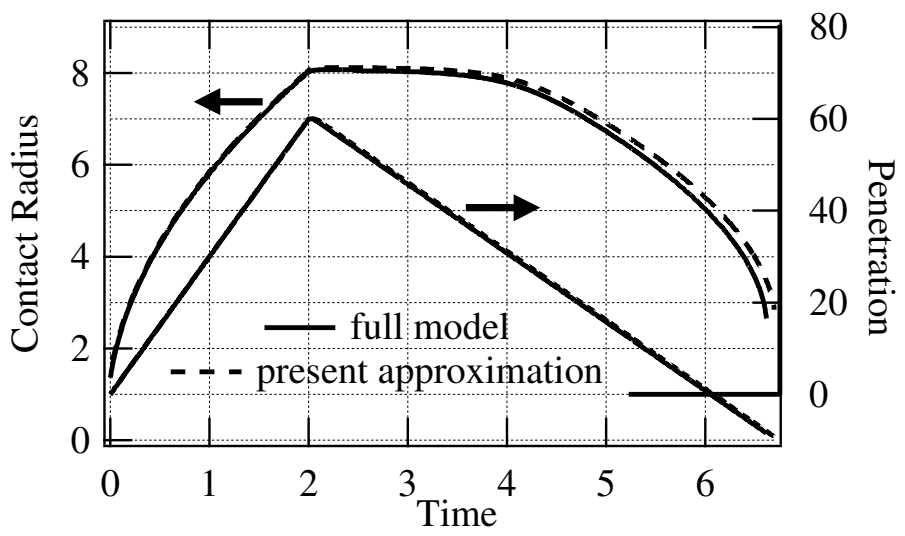

Figure 5: 


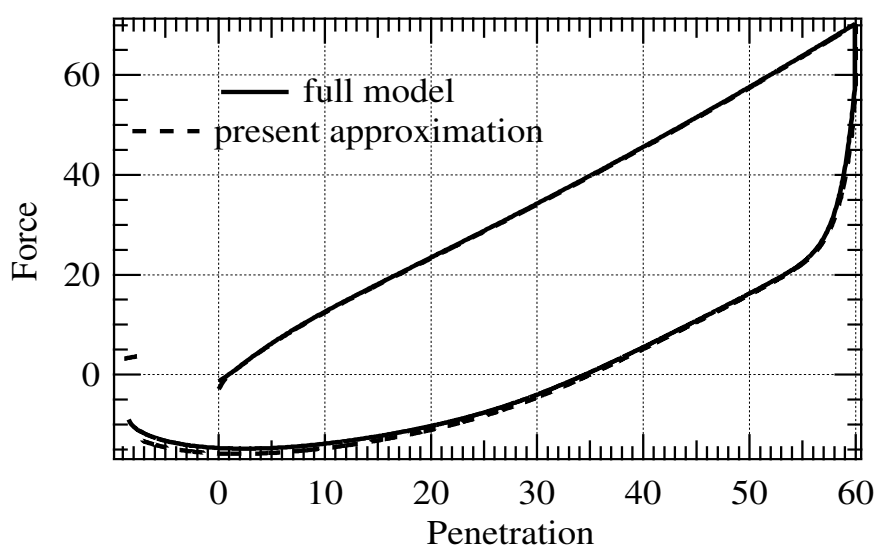

Figure 6:

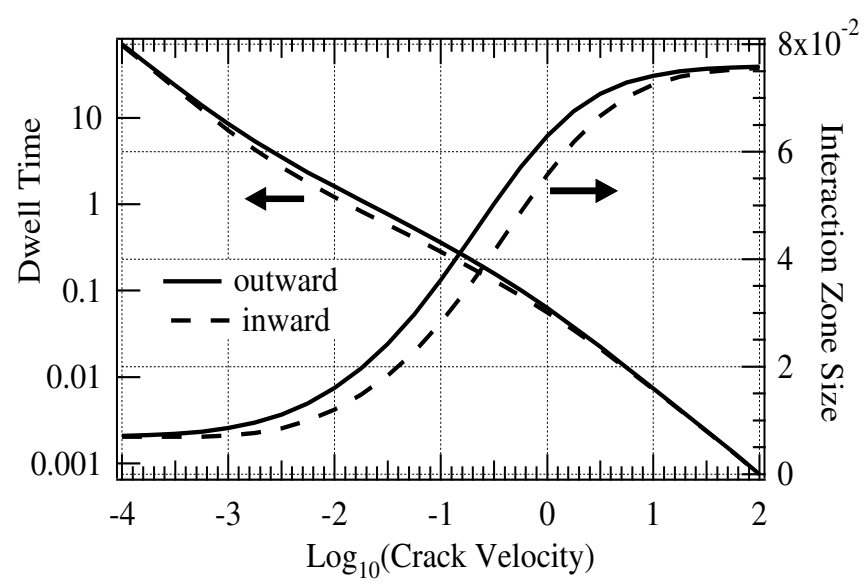

Figure 7: 


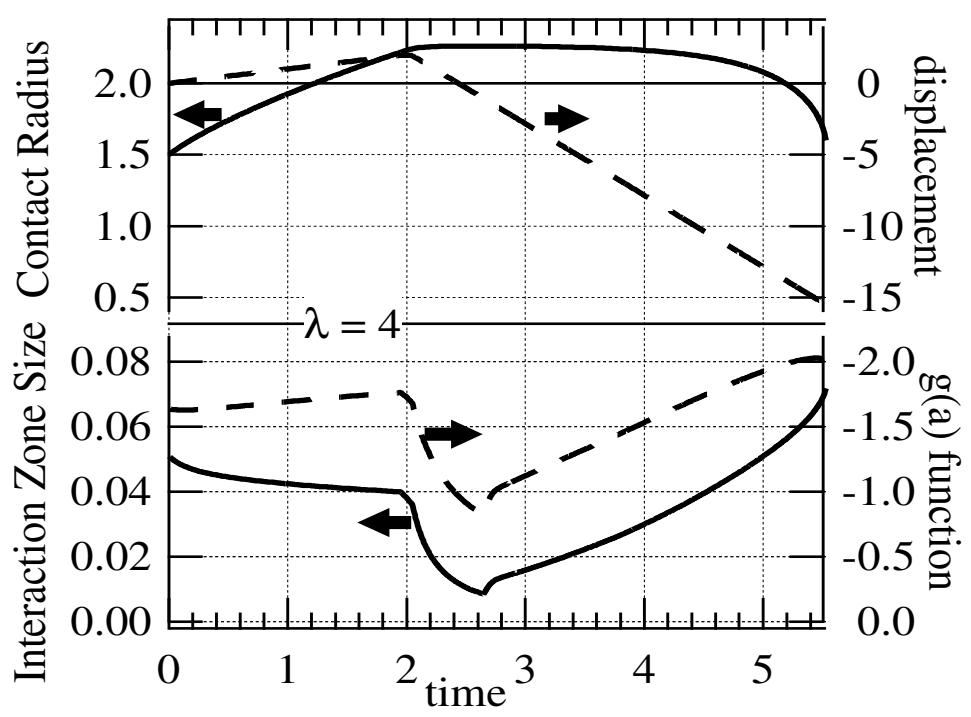

Figure 8:

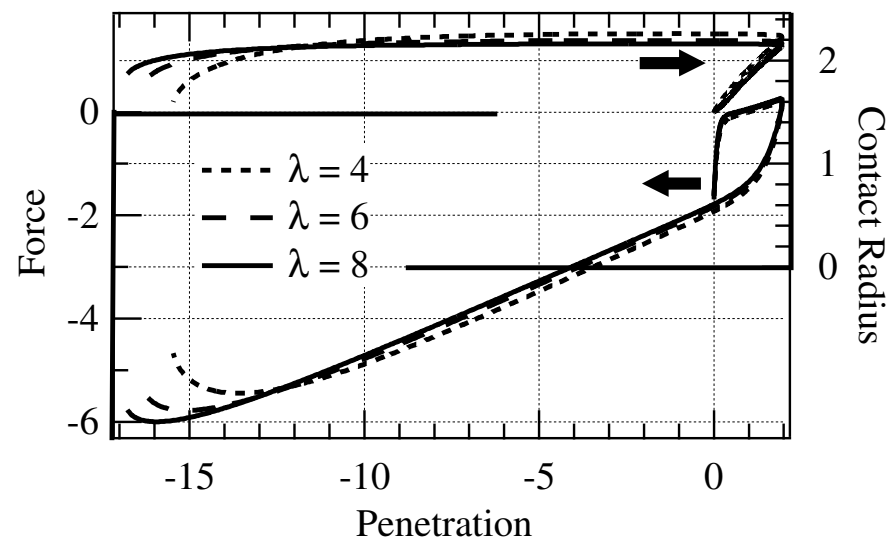

Figure 9: 